\title{
A conceptual framework to compare two paradigms of Augmented and Mixed Reality experiences
}

\author{
Laura Malinverni, Cristina Valero, Marie-Monique Schaper, Narcis Pares \\ Universitat Pompeu Fabra \\ Barcelona, Spain \\ malinverni.laura@gmail.com, crisvaleroharo@gmail.com, mariemonique.schaper@upf.edu,
narcis.pares@upf.edu
}

\begin{abstract}
Augmented and Mixed Reality mobile technologies are becoming an emerging trend in the development of play and learning experiences for children. This tendency requires a deeper understanding of their specificities to adequately inform design. To this end, we ran a study with 36 elementary school children to compare two AR/MR interaction paradigms for mobile technologies: (1) the consolidated "Window-on-the-World" (WoW), and (2) the emerging "World-as-Support" (WaS). By analyzing children's understanding and use of space while playing an AR/MR mystery game, and analyzing the collaboration that emerges among them, we show that the two paradigms scaffold children's attention differently during the experience and elicit different forms of collaboration. We conclude by presenting a conceptual framework to distinguish the strengths, weaknesses, and potential of the two AR/MR paradigms, as well as the comparison between marker-based and marker-less technical solutions. This study aims at helping practitioners in taking design decisions for AR/MR technologies for children.
\end{abstract}

\section{Author Keywords}

Mixed Reality; Augmented Reality; embodied interaction; embodied cognition; meaning making; Window-on-theWorld; World-as-Support.

\section{ACM Classification Keywords}

H.5.1. Information interfaces and presentation (e.g., HCI): Multimedia Information Systems

\section{INTRODUCTION}

Augmented and Mixed Reality (AR/MR) mobile technologies offer the evocative potential of making the

Permission to make digital or hard copies of all or part of this work for personal or classroom use is granted without fee provided that copies are not made or distributed for profit or commercial advantage and that copies bear this notice and the full citation on the first page. Copyrights for components of this work owned by others than ACM must be honored. Abstracting with credit is permitted. To copy otherwise, or republish, to post on servers or to redistribute to lists, requires prior specific permission and/or a fee. Request permissions from Permissions@acm.org.

IDC '18, June 19-22, 2018, Trondheim, Norway

(C) 2018 Association for Computing Machinery.

ACM ISBN 978-1-4503-5152-2/18/06 \$15.00

https://doi.org/10.1145/3202185.3202750 invisible visible and adding new layers of meaning to our physical world. These features, in the context of ChildComputer Interaction, open the path for emerging possibilities related to creating novel relations of meaning between the digital and the physical, between what can be directly seen and what needs to be discovered, between our physical surroundings and our imagination.

This potential has attracted both research and industry, which are increasingly designing mobile-based AR/MR technologies to support a wide variety of play and learning experiences. This emerging trend has shaped AR/MR as one of the leading emerging technologies of 2017 [16], hence requiring practitioners to face novel ways of thinking about mobile technologies and designing for them. Consequently, a deeper and fuller understanding of AR/MR specificities is required to design systems capable of taking advantage of its affordances and potential. Furthermore, additional considerations should address how different AR/MR design choices can be more suitable to support specific kinds of play and learning experiences for children.

Starting from this perspective, the current research aims at digging deeper into the understanding of AR/MR mobile technologies for children to offer guidelines for researchers and practitioners. Specifically, we will focus on examining and comparing two $\mathrm{AR} / \mathrm{MR}$ paradigms for mobile technologies: (1) the consolidated "Window-on-the-World" (WoW) interaction paradigm which is based on the screen of a mobile device to merge computer-generated images with the user's view of the physical surroundings and (2) the emerging "World-as-Support" (WaS) interaction paradigm [10] which proposes the use of projective technologies to project computer-generated images directly on the physical environment.

To compare these two paradigms, we will first overview AR/MR mobile technologies for children and introduce the specificities of the two examined paradigms. Subsequently, we will present a study aimed at investigating how the WoW and WaS paradigms can shape children's understandings, collaboration and use of space in a task based on playing an AR/MR mystery game. By contrasting the use of the same application with two devices (one based on the WaS and one based on the WoW), we will show how the two paradigms 
had different implications on how children allocated their attention during the experience and elicited different forms of collaboration.

To conclude, we build on the results of the study and on previous research in this field to propose an initial conceptual framework aimed at helping practitioners in making design decisions (and defining research questions) to support specific kinds of play and learning experiences with AR/MR technologies for children.

\section{AUGMENTED AND MIXED REALITY FOR CHILDREN}

Augmented/Mixed Reality (AR/MR) technologies provide compelling experiences that allow users to interact with the physical world and computer-generated content. The recent advances in the technical capabilities of smartphones and tablets have spurred the interest in $\mathrm{AR} / \mathrm{MR}$ solutions for mobile technologies [5]. This is making them easily accessible and very popular both in the research community as well as in everyday experience of users. On the one hand, specific frameworks are being developed to support the creation of AR/MR applications (e.g. Google ARCore, Apple ARKit). On the other, both academy and industry are increasingly exploring the potential of using AR/MR solutions in different fields such as: education [7], creativity [24], gaming [1], and cultural heritage [15,19]. This panorama has shaped AR/MR solutions as one of the leading emerging technologies of 2017 [16], hence requiring a specific effort to fully understand its specificities, affordances and potential.

In the context of Child-Computer Interaction, AR/MR technologies have been employed to support a wide variety of play and learning experiences. Examples can be found in: augmented reading and coloring books (e.g. [4,25]); AR videogames (e.g. Pokemon Go); applications that allow augmentation and addition of multimedia contents to curricular materials (e.g. [26]); systems that use AR to support learning while visiting cultural heritage sites (e.g. $[7,19])$; and applications that allow children to create their own AR contents (e.g.[27]).

This diverse panorama encompasses a wide variety of technological solutions and a miscellaneous experiential goals. Some relevant distinctions can be drawn in relation to: (1) the goals pursued by the application; (2) the social context for which the AR/MR activity is designed; and (3) the relation that the system establishes with the physical world. In relation to the pursued play and learning goals, applications that allow children to create their own AR contents mainly target open and creative activities (e.g. Aurasma). Instead, those that augment curricular materials mainly address the transmission of specific contents. Similarly, in relation to the social context, for instance, augmented reading books mainly aim at supporting individual amusement experiences. Instead, the systems that use AR to support learning are mainly designed for a collaborative and shared use (e.g. [7,19]). Finally, different designs propose different kinds of relationships between the users and the physical world. For instance, some applications do not offer, or require a tight coupling with the physical world (e.g. the location of Pokemons in Pokemon Go does not carry any specific meaning beyond its playability). Other need to be used in a specific site to acquire their full meaning (e.g. Augmented cultural heritage experiences, which are only meaningful at the specific site).

Besides reflecting the richness of opportunities, the diversity of AR/MR-based experiences asks for a better understanding of how different configurations can support our intended play and learning goals. Starting from this perspective, we examine a consolidated and an emerging paradigm in AR/MR mobile technologies to better understand how they can mediate user experience and respond to different purposes, goals and specificities.

\subsection{Consolidated and emerging paradigms in AR/MR technologies: The Window-on-the-World and the World- as-Support}

From a technological perspective, most AR/MR applications for children are generally designed according to the Window-on-the-World (WoW) interaction paradigm. This paradigm represents a well-known approach to make the digital and the physical worlds come together. It finds its roots in early works on AR [12] and, technically, it is based on using the display of a mobile device to merge computergenerated images with images captured from the physical surroundings by the camera of the mobile device. This effect allows users to view virtual elements on the physical world and eventually interact with them to enable specific functionalities [14].

The limited hardware requirements of the WoW have transformed this solution in the dominant paradigm of AR/MR applications. Nonetheless, as Betsworth et al. [3] and Müller at al. [14] pointed out, this paradigm can present some relevant limitations. First, by requiring users to hold their devices in front of the physical world, the system runs the risk of becoming a "digital divider" [3]. Second, the prominence of the digital content may reduce the attention that users pay to the physical environment during the experience [3].

To respond to these limitations, novel technological solutions are being developed. From the review of the state of the art in the field of HCI, we identified the rise of a new AR/MR mobile-based technological configuration. We analyzed this configuration conceptually and defined its specific potential. We decided to call this novel approach the "World-as-Support" (WaS) interaction paradigm and formalized it in Malinverni et. al [10]. From a technical perspective, the WaS paradigm is grounded in the tradition of Projective Augmented Reality and Spatial Augmented Reality [18]. These technologies allow the dynamic recognition of the physical world (i.e. geometry, surfaces, objects and movements) and the projection of context-aware information directly onto it. This enables users to project contents in their surroundings and use the physical world to 
interact with these contents. Examples can be found in research by $[3,13,21]$. For instance, Molyneaux et al. [13] present a system that combines pico-projectors with Kinect and IR cameras to create high-quality $3 \mathrm{D}$ models of the physical world and project context-aware digital contents on it. Instead, Betsworth et al. [3] use a simplified system based on QR codes to project the digital information directly onto the physical elements of a heritage site. Finally, Willis et al. use pico-projectors, motion sensors and a camera-based vision system to animate the motion of virtual characters [21], enhance storytelling experiences [22], and promote side-by-side multi-user gaming interactions [23].

To sum up, current research in AR/MR mobile technologies proposes different technological solutions (WoW and WaS) which do not only represent different hardware configurations, but also, and more importantly, offer different ways of constructing and shaping user experience. We do not mean to say that one paradigm should be considered better than the other. Instead, it implies that the two paradigms offer specific strengths, affordances and weaknesses that require to be fully understood to define the most appropriate design choices in the development of AR/MR technologies for children.

\section{Previous research on the WaS paradigm for children applications}

The present study is part of an ongoing project dedicated to better understand the potential of the WaS interaction paradigm for designing $\mathrm{AR} / \mathrm{MR}$ mobile applications for children. Within this project, we carried out two initial studies aimed at investigating how this paradigm can mediate user experience, meaning making and social interaction.

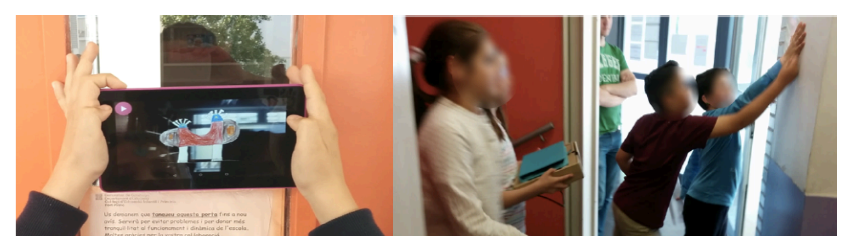

Figure 1. From left to right, child playing with the WoW and children playing with WaS.

In the first study [10], we contrasted the potential of the WoW and WaS paradigms in an AR/MR application aimed at supporting the collaborative improvisation of site-specific narratives by children. The children were provided with one of two low-fidelity prototypes: one based on the WoW and the other on the WaS paradigms. They started by creating their own fictional characters. They were then asked to use one of the two AR/MR systems to situate the characters in the physical space of their school and invent collaborative fictional narratives about them (Figure 1). The results from the study suggested that the two paradigms supported different ways of engaging and using the physical/digital space in interactive experiences. Specifically, the WaS paradigm, allowed children to explore and interact more with physical space and use it as a narrative element in their stories. At the same time, the two paradigms, strongly affected the unfolding of social relationships and instances for participation and co-construction of meaning. Namely, children in the WaS condition participated more as coauthors of their peers' stories and paid more attention to stories told by others. Instead, in many cases, the children using the WoW showed only a limited participation with and attention on the others.

In the second study, we explored the potential of the WaS paradigm to design site-specific cultural heritage experiences [20]. We designed an educational experience for a bomb shelter dating from the Spanish Civil War, known as Refugi 307. We followed a co-design approach to analyze the project requirements and include the different needs and viewpoints of the involved stakeholders (students, teachers, curators, guides and designers) in the design. The prototype of the WaS experience was based on a marker-recognition system. We used the markers strategically to trigger contents in specific locations of the shelter in a simple manner. The project's goal was to complement the current guided visit with the aim of: (1) increasing students' context awareness between specific physical aspects and historical events; and (2) strengthening the understanding of historical contents that require emotional engagement and critical thinking. The outcomes based on a study with primary school students showed that projecting and interacting with digital content within the shelter helped them to better imagine how civilians experienced the bombing raids during the Spanish Civil War (Figure 2).

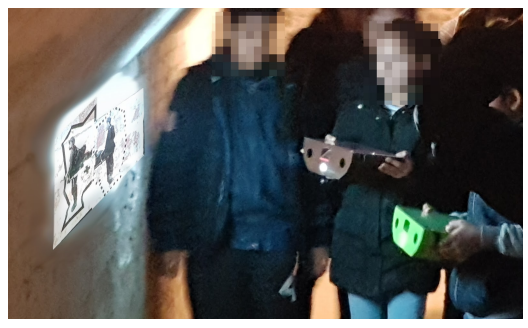

Figure 2. Children using the WaS in the Refugi 307 project

These previous studies propose different ways of using the WaS paradigm, which respond to the specific goals of each application (i.e. creative narrative improvisation vs. learning about cultural heritage). Building on the findings of this previous research, the presented study aims at deepening into the research on the $\mathrm{WoW}$ and $\mathrm{WaS}$ paradigms in order to extrapolate generalizable knowledge and inform designers and practitioners interested in AR/MR mobile solutions.

\section{THE STUDY}

The present study had the goal of investigating how the WoW and WaS paradigms can shape children's understandings, collaboration, and use of space in a task based on playing with an AR/MR mystery game based on a fictional story. Its ultimate objective is to delve into the research on how these paradigms can be employed to design $\mathrm{AR} / \mathrm{MR}$ applications for children. In the following sections, we describe the employed application, the prototypes for the 
two AR/MR systems, the procedure, and data analysis of the study.

\subsection{The Application}

The study used an application called "Saint George's Dragon". This application represents a fictional story of two dragons (the protagonists) who find that somebody has painted graffiti on the walls of the school reception during the night. The mission of the children was to discover who painted those graffiti. The suspects are five well known characters from classical children stories, who escaped from their books the same night that the graffiti appeared. The children were encouraged to help the dragons to discover who had painted every graffiti. The story was presented to the children by the application itself. The experience was divided into two stages as described below.

First, the experience started with an animation of the dragons introducing the story to the children and asking them to help them solving the mystery. Subsequently, the children had to find five physical markers located around the walls of the reception area of the school (Figure 3). Each marker was associated to one graffiti, and when children pointed at them with the device, the virtual graffiti was projected just below its corresponding marker. After finding all the graffiti, the children moved onto the second part of the experience. The second stage was located in the school library. Here, the children found five other markers. In this stage, by pointing at a marker with the device, the children obtained the projection of a dialogue between the dragons and one of the five suspect story characters. After having listened to all the dialogues, the children were encouraged to discuss between them who they thought had painted the graffiti on the reception walls. The application was developed in two different formats using the WoW interaction paradigm for one and the WaS for the other.

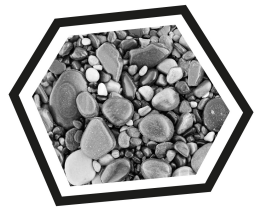

Figure 3. Example of a marker

\subsection{The Prototypes}

For the study, we used the following devices to compare the WaS and the WoW paradigms:

1. The WaS system: the device recognizes markers in physical space and projects virtual content next to the marker on any type of surface. It is composed of an Android Aquaris BQ tablet, a Bluetooth loudspeaker, and a pico-projector. All components are enclosed in a specially designed casing for our prototype (Figure 4).

2. The WoW system: the device recognizes markers in physical space and displays, through its screen, virtual content overlaid on images captured directly from physical space, according to the classic AR configuration. To this end, we used an Android Aquaris BQ tablet (Figure 4).

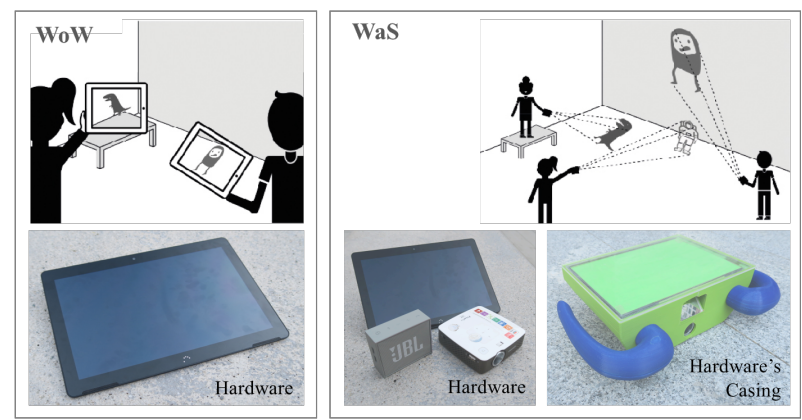

Figure 4. The configuration of the WoW system (left) and of the WaS system (right)

\subsection{The Procedure}

The study was carried out with 36 children (14 boys and 22 girls; between 9 and 11 years old) of two 4th grade classes from a local school in Barcelona. To compare the two paradigms, we used a between-subjects design. The class was divided into groups of 4 to 5 children. The groups were decided by the children under the consent of the teacher. Subsequently, each group was randomly assigned to either the WaS condition (WaSc) or to the WoW condition (WoWc). A total of 8 groups were created (4 WaSc and 4 WoWc).

To start the activity, one researcher informally asked the children about their experience with AR. Almost all children were familiar with AR apps such as Pokemon Go. Hence, the researcher explained them that they would participate in a study using AR. Subsequently, two groups of the children belonging to the same condition (either WaSc or WoWc) were led to the reception area. Once there, one researcher explained that we had created a special story for their school. Specifically, she explained that, to be able to enjoy the story, they needed to use the device and point at the "magical portals" (the markers) located on the walls of the space. The researcher explained that they had to resolve a mystery. Therefore, we recommended them to pay attention to the clues found in the story description and dialogues. Finally, we gave one device to each group and we led them to the first marker. The children were not instructed on how to share the device between them and they were allowed to organize the use of the device according to their preferences.

After pointing their device at the first marker and listening to the protagonists explaining the story introduction, the children could start to explore the "graffiti markers". When the group had found, and explored all markers in the reception, the researcher encouraged them to think where they could go to look for the story characters. If they did not come up with a solution, the researcher suggested to them to go look in the library. The children then explored the markers in the library and listened to the dialogues between the dragons and the story characters. We then gave the children 5 minutes to discuss and decide who they thought had 
painted the graffiti on the reception walls. To do that, the children were asked to sit around a table in the library.

Subsequently, we took the children back to their classroom. We gave each one a blank sheet of paper and asked them to individually draw what they had liked the most about the activity. Finally, 10 children (randomly selected between the two conditions; 5 and 5) were interviewed by two researchers. The interview was done back in the library and focused on asking the children to explain their drawings. Specifically, we followed a semi-structured approach that started from the drawing and investigated the context and motivation behind it. Examples of the questions we used include: "What did you draw? Why? What was happening at this moment? Where were you? Who was there with you? What were they doing? How did you start? And what did you do later? How? How did you finish?". The overall activity (experience and interviews) lasted for approximately 2 hours and was video recorded by two researchers using two handheld cameras.

\subsection{Data Analysis}

To research how the WoW and WaS paradigms shaped children experience in the Dragon's story, we triangulated data from the analysis of the video recordings of their in situ interactions during the Dragon's activity; the analysis of the drawings; and the analysis of the interviews.

\section{Analysis of in situ interaction}

The analysis of children's in situ interactions during the Dragon activity focused on researching how they interacted with the system, how they used and moved in physical space, how they interacted between them, and how they organized the collaborative task. To this end, we employed a multimodal analytical approach based on the model proposed by $[8,11,17]$. This approach is based on acknowledging that people employ multiple resources (e.g. verbal, visual, kinesthetic, aural, etc.) to construct and express meaning. Therefore, it proposes methodological tools and techniques to include these multiple resources in the analysis of the meaning making process.

Following this approach, two researchers first watched all the videos of the interaction of children in situ, to get a general view of the experience. Due to their long duration and the homogeneous children's behavior, we agreed to confine the multimodal analysis on the children's interactions with three (out of the five) markers from the reception, and three (out of the five) markers from the library, per group. Due to a technical problem with the devices, two groups (1 WaSc; 1 WoWc) were excluded from the analysis.

Subsequently, the two researchers together visualized the selected videos in slow-motion several times to transcribe and analyze the resources employed by the children during exploration and interaction. To carry out this analysis, we followed the model proposed by Malinverni et al. [11] and focused the following resources: experimentation with the virtual objects; use of space; gestures; social interactions; group distribution; interaction with the device; gaze directionality. These resources were transcribed in a tabular format for the subsequent interpretation and triangulation.

\section{Analysis of children's drawings}

To analyze the drawings, we employed a mixed approach that combined content analysis and multimodal analysis. First, two researchers together reviewed all the drawings to define a set of grounded categories based on the elements that were included in the drawings (see table 1 for a summary of the identified elements). Then, each researcher analyzed half of the drawings to count the elements that were included in each drawing.

\begin{tabular}{|l|l|}
\hline Markers & The child draws the physical marker \\
\hline Device & The child draws the device \\
\hline $\begin{array}{l}\text { Props belonging to } \\
\text { physical space }\end{array}$ & $\begin{array}{l}\text { The child draws any physical elements } \\
\text { of real space where the action took } \\
\text { place. }\end{array}$ \\
\hline Virtual element & $\begin{array}{l}\text { The child draws any virtual elements of } \\
\text { the story }\end{array}$ \\
\hline First person agent & $\begin{array}{l}\text { The child draws himself carrying the } \\
\text { device }\end{array}$ \\
\hline Peers & The child draws his peers \\
\hline
\end{tabular}

Table 1. Categorization of the elements included on the drawing

After this preliminary activity, we analyzed the drawing according to a multimodal approach based on Kress' theoretical framework [9]. This analysis was oriented toward interpreting what were the most important elements of the drawings. To this end, we analyzed the layout of the drawings; the relative arrangement, relative size and position of the elements in the drawings; and the level of details [9]. With this analysis, we identified four main categories that describe what elements had received most attention in the drawings:

- The overall context: the overall scene is depicted, including physical elements, peers, markers, devices, etc. (Figure 5, first).

- The device: the device occupies most of the space of the drawing (Figure 5, second).

- $\quad$ The marker: the marker occupies most of the space of the drawing (Figure 5, third).

- The virtual element: a virtual element occupies most of the space of the drawing (Figure 5, fourth).

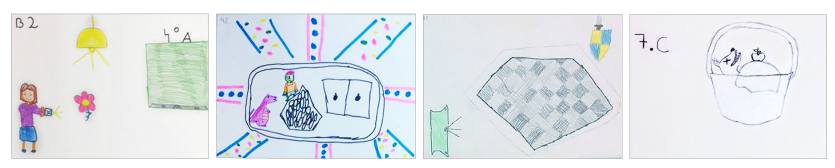

Figure 5. Examples of drawings from each category

\section{Analysis of the interviews}

To analyze the interviews, we employed a content analysis approach. Specifically, one researcher completely transcribed the interviews. Then, the transcripts were analyzed focusing on: (1) The elements that the children 
included in their description of the experience; (2) the verbs that children used to describe the experience.

Data from the analysis of in situ interactions, drawings, and interviews, were finally merged for interpretation.

\section{RESULTS}

In this section, we will first report on the results related to the different sources (in situ interaction, drawings, interviews, and questionnaires). Subsequently, we will present the main findings derived from their triangulation.

\subsection{In situ interactions}

To analyze the children's in situ interactions, we first focused on their embodied interactions with the physical environment and the device by observing their use of space and the spatial arrangement of the group. We then analyzed their reciprocal interactions by observing the gestures that they intuitively employ, their verbal interactions and their ways of organizing the collaborative activity (e.g. the role that they assumed).

The children did not use or experiment with the physical space in any of the two conditions. Instead, they mainly went from marker to marker. None of them used the space to experiment with different options of visualizing the content (e.g. projecting the content on different surfaces), or to physically interact with it. Specifically, in the WaSc the children did not project the characters onto the space, or touched or interacted with any spatial element. Similarly, in the WoWc the children did not look at the space unless there was a marker in front of them to trigger the digital content on the tablet screen.

Although the way in which they used space were similar in both conditions, we observed relevant differences related to the spatial arrangement of the groups during the activity. In the WaSc, the children kept a semi-circular disposition around the device all the time. Instead, in the WoWc, the children changed their group disposition depending on the different stages of the experience (reception or library). Specifically, in the reception, the group disposition was scattered. Instead, in the library, the groups assumed a semicircular disposition around the device. This change was mainly due to the fact that in the library, the dialogues between the animated virtual characters required children to pay attention to their talk.

In both conditions, the children showed both verbal and nonverbal interactions oriented toward organizing the collaborative activity. Children's gestural interactions mainly covered the functions of: (1) indicating their peers to look at the virtual elements or the next marker through deictic gestures; (2) regulating their reciprocal interactions (e.g. asking for silence to be able to listen to a member of the group or to the virtual character). Similarly, their verbal interactions mainly focused on: describing the virtual elements or making comments on them (8 times in WaSc, 7 WoWc); or regulating their reciprocal interactions (5 times in WaSc, 6 WoWc). Despite these similarities, however, some important differences were observed between the two conditions. Specifically, in the WaSc, the children performed more gestures to express emotion or surprise for the appearance of the characters ( 5 times) with respect to WoWc (only 1 time). Furthermore, in the WASc, the children showed more verbal interaction oriented toward coconstructing knowledge with respect to the WoWc (6 times in WaSc, 1 in WoWc): e.g. sum up their findings, contrast hypothesis about the mystery.

This latter difference was deeply related to the roles that the children assumed in the two conditions. In the WaSc, the children did not have clearly differentiated roles and all moved around together. Instead, in the WoWc, the groups established a clear role division; while one child was in charge of controlling the device, another explained what had appeared in the device to a third child who took notes but did not necessarily look at the digital content.

\subsection{Drawings}

In both conditions, the most recurrently depicted elements were: the virtual characters (WoWc: 13; WaSc: 15), the markers (WoWc: 8; WaSc: 13), and the devices (WoWc: 9; WaSc: 12). To compare the two conditions, we first ran a chisquare test for each category. Results showed no significant differences between the two conditions for almost all categories (Figure 6) except for the "first person agent" category. What this means is that, the children in the WaSc tended to represent themselves in their drawings more as first person acting agents, compared to children in the WoWc groups $(\mathrm{WaSc}=9 ; \mathrm{WoWc}=1 ; \chi 2(1)=7.49, \mathrm{p}<.05)$.

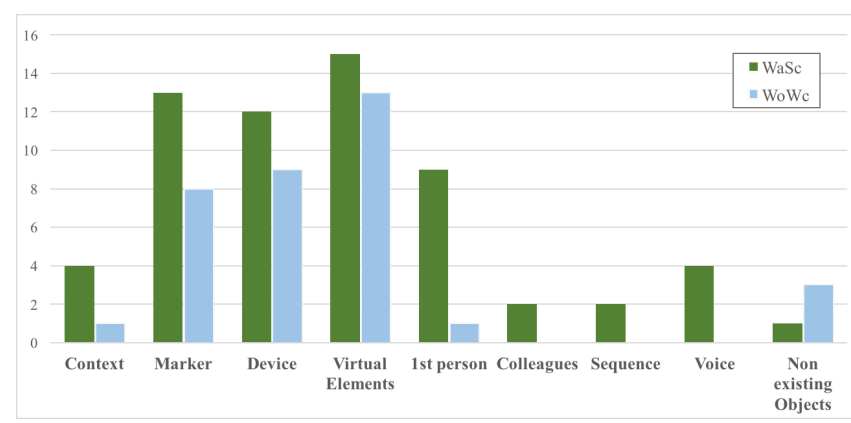

Figure 6. Comparison of the elements depicted in the drawings between WoWc and WaSc

Subsequently, we calculated the diversity index of each drawing (i.e. number of different elements depicted in the drawing / number of total elements) and compared the two conditions using a t-test. Results showed that the drawings belonging to the WaSc $(\mathrm{M}=0.49 ; \mathrm{SD}=0.2)$ had a significantly higher diversity of drawn elements compared to the WoWc $(\mathrm{M}=0.3 ; \mathrm{SD}=0.12), \mathrm{t}(27)=-3.01, \mathrm{p}<.05$. This means that the children in the WaSc chose to represent the experience by including a much higher number of elements (i.e. props belonging to the physical space, peers, etc.) compared to the children in the WoWc who mainly focused on depicting the markers, the device and the virtual characters.

The tendency of the children is the WaSc to give a more holistic representation of the experience was particularly evident in the multimodal analysis of the most important 
elements of the drawings. Using the classification described in section 3.4 (context, device, marker and virtual element), we ran a chi-square test for each category to compare the two conditions. Results showed significant differences between the WoWc and the WaSc (see Figure 7). Specifically, the children in the WaSc tended to give more importance to the general context of the experience, in contrast to children in the WoWc, by depicting their spatial location, furniture, etc. $\left(\mathrm{WaS}=8 ; \mathrm{WoW}=2 ; \chi^{2}(1)=4.88, \mathrm{p}<.05\right)$ Instead, the children in the WoWc tended to give more importance to the device by drawing it large, central, and very detailed $(\mathrm{WaSc}=1 ; \mathrm{WoWc}=7 ; \chi 2(1)=6.80, \mathrm{p}<.05)$.

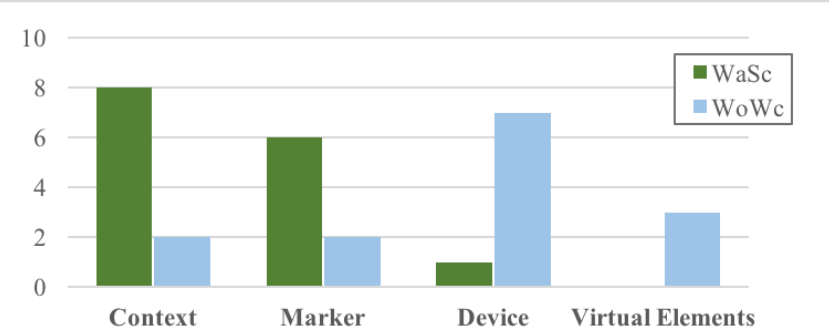

Figure 7. Comparison of the elements they give importance to between WoWc and WaSc

\subsection{Interviews}

To analyze the interviews, we first quantified the elements that the children mentioned in their description of the experience and the verbs that they used to describe their actions and roles. We, then, analyzed their understandings of these concepts.

In both conditions, the children performed a similar amount of verbalizations, calculated according to the number of used words (WaSc: $\mathrm{M}=141, \mathrm{SD}=71,56$; WoWc: $\mathrm{M}=148,4, \mathrm{SD}=$ $36,7)$. Similarly, in both cases, they tended to speak mainly about the virtual characters (33 verbalizations; $20 \mathrm{WaSc} ; 13$ WoWc), the device (30 verbalizations; $14 \mathrm{WaSc} ; 16 \mathrm{WoWc}$ ), and the markers (23 verbalizations: $14 \mathrm{WaSc}$; 9 WoWc). Instead, verbalizations on the physical space were rather scarce (10 verbalizations; $6 \mathrm{WaSc} ; 4 \mathrm{WoWc})$ and, in all cases, they referred only to the general context (e.g. "we were in the reception / library") without mentioning any specific object or feature (Figure 8).

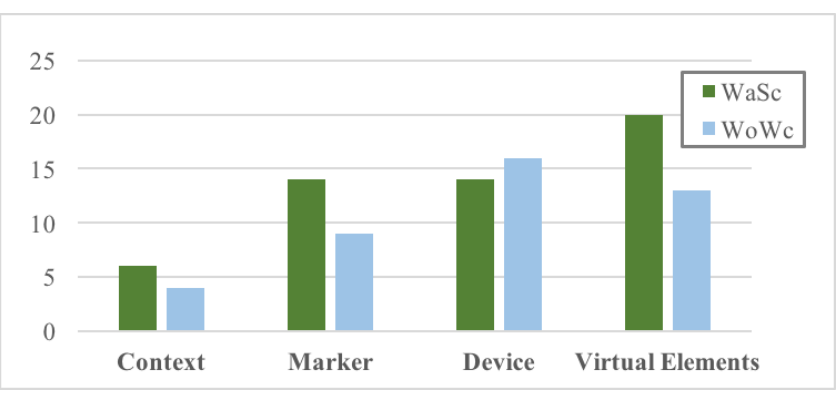

Figure 8. Comparison of the elements mentioned during the interview

By running a t-test, we found significant differences between the two conditions in the number of verbalizations about "virtual characters" and "markers". Specifically, the children in the WaSc $(\mathrm{M}=4 ; \mathrm{SD}=1)$ tended to mention more often the virtual characters than the children in the WoWc $(M=2.6$; $\mathrm{SD}=0.9), \mathrm{t}(8)=2.33, \mathrm{p}<.05$. In both cases, the verbalizations on the virtual characters mainly referred to finding them (e.g. "and then we found the Puss in Boots"), or to something that the characters had said. Similarly, the children in the WaSc $(\mathrm{M}=2.8 ; \mathrm{SD}=0.48)$ mentioned more often the markers than the children in the WoWc $(\mathrm{M}=1.4 ; \mathrm{SD}=0.55), \mathrm{t}(8)=4.42, \mathrm{p}$ $<.05$. Their understandings of the markers were mainly framed around their functional role in allowing them to enjoy the experience (e.g. "When you placed the tablet on top of the 'paperboard', a character appeared"). Nonetheless, it is relevant to notice that in 6 cases $(5 \mathrm{WaSc} ; 1 \mathrm{WoWc})$, the children explicitly referred to the role of the markers in shaping the spatial and temporal organization of the experience (e.g. "We had to go through each image to see if it was this or that"; "We were doing this first and later that").

The differences in their understandings of the experience were particularly evident in the analysis of the action verbs that they employed to describe their activities. Specifically, we identified the following action verbs as the most recurrent: find/look for ( $8 \mathrm{WaSc} ; 14 \mathrm{WoWc})$; think about / reflect on (13 WaSc; 8 WoWc); watch ( 8 WaSc; 3 WoWc); move (9 WaSc; 2 WoWc) (Figure 9)

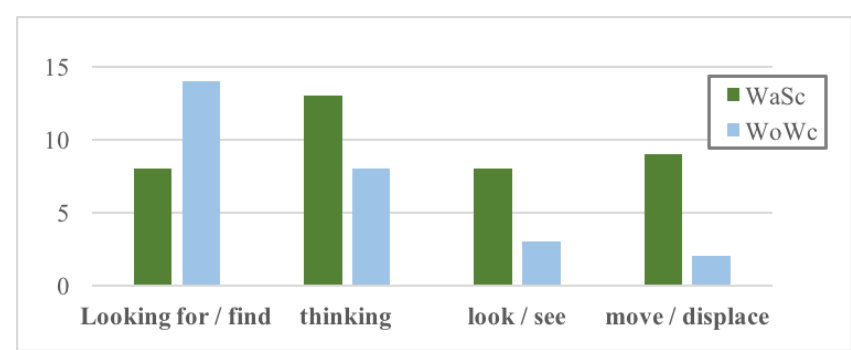

Figure 9. Comparison of action verbs employed during the interview

By running a t-test, we found significant differences for all verb categories (Table 2). From these results, it is relevant to notice that the children in the WoWc associated the experience mainly with the task of having to find something. Instead, the children in the WaSc used a much higher variety of action verbs (think about / reflect on; watch; move).

\begin{tabular}{|l|l|l|l|}
\cline { 2 - 4 } \multicolumn{1}{c|}{} & WaSc & WoWc & $\begin{array}{l}\text { Independent sample } \\
\mathrm{t} \text {-test }\end{array}$ \\
\hline $\begin{array}{l}\text { Find / } \\
\text { lookfor }\end{array}$ & $\begin{array}{l}\mathrm{M}=1.6 \\
\mathrm{SD}=0.55\end{array}$ & $\begin{array}{l}\mathrm{M}=2.8 \\
\mathrm{SD}=0.48\end{array}$ & $\mathrm{t}(8)=-3.79, \mathrm{p}<.05$ \\
\hline $\begin{array}{l}\text { think about / } \\
\text { reflect on }\end{array}$ & $\begin{array}{l}\mathrm{M}=2.6 \\
\mathrm{SD}=0.55\end{array}$ & $\begin{array}{l}\mathrm{M}=1.6 \\
\mathrm{SD}=0.55\end{array}$ & $\mathrm{t}(8)=2.88, \mathrm{p}<.05$ \\
\hline watch & $\mathrm{M}=1.6$ & $\mathrm{M}=0.6$ & $\mathrm{t}(8)=2.88, \mathrm{p}<.05$ \\
\hline move & $\mathrm{SD}=0.55$ & $\mathrm{SD}=0.55$ & $\mathrm{M}=1.8$ \\
$\mathrm{SD}=0.84$ & $\begin{array}{l}\mathrm{M}=0.4 \\
\mathrm{SD}=0.55\end{array}$ & $\mathrm{t}(8)=3.13, \mathrm{p}<.05$ \\
\hline
\end{tabular}

Table 2. Comparison of action verbs 


\subsection{Triangulating data sources}

The analysis of the different sources (in situ interactions, drawings, interviews) offered a consistent panorama of how the two interaction paradigms (WoW and $\mathrm{WaS}$ ) mediated children's experiences and understandings of an AR/MR playful fictional story.

How children understand and relate virtual contents and the physical world.

In the WoWc, the children's representations of the experience (drawings and interviews) were mainly focused on the relation between the device, the marker, and the virtual elements, where crucial importance is given to the device. This tendency can be observed in their drawings, where the device becomes the central focus of attention, and in their verbal explanations that mainly described the act of finding the contents hidden behind the markers. Conversely, in WaSc, the children seem to have a more holistic understanding of the experience by including the context as a relevant part of it. This tendency can be seen in their choice of drawing the overall setting of the experience; including a wider diversity of elements in their drawings (e.g. props belonging to the physical space, peers, etc.); and using a broader variety of action verbs to describe the activity. This difference could be explained in relation to the space where the virtual experience took place in the two conditions. In the WoWc, the experience is communicated to the children only through the screen of the tablet device and the physical space is only perceived as an image backdrop on the screen with the virtual elements overlaid on it. Instead, in the WaSc the virtual elements are projected onto the physical world, which asks the children to actively and directly look at the physical space.

Nonetheless, even when the children in the WaSc seem to pay more attention to the physical context than children in the WoWc, their understandings of space mainly refers to the general context where the activity took place (e.g. "we were in the reception / library"). Furthermore, in both conditions, the children did not consider space as something with which they can interact. From the analysis of in situ interaction, we observed that no group used or experimented with the physical space. Instead, they went mainly from marker to marker. In both conditions, the markers worked as landmarks that discretized physical space into isolated units. In other words, what really mattered to the children in the experience occurred only where the markers were present, hence making the rest of the space not worth being explored. This is shown in the prominent role of the markers in children's drawings ( $72,41 \%$ of drawings included markers) and in their verbalizations, where 6 out of 10 children explicitly referred to the role of the markers in shaping the spatial and temporal organization of the experience. These findings are indicative of how the interplay between the used device and the markers shaped their ways of understanding the experience, using the space, and building a relation between the device, the virtual contents, and the physical world.

\section{How children organize social interactions}

In both conditions, the children collaborated between them in the task and actively shared their thoughts. Nonetheless, some subtle differences were observed in their social interaction patterns. In the WoWc, the children showed a scattered group disposition and tended to establish a clear division of roles between them. Instead, in the $\mathrm{WaSc}$, they did not have a clear role division and tended to move around all together all the time. Furthermore, in WaSc, the children showed more verbal interaction oriented toward coconstructing knowledge compared to the WoWc, and used more verbs related to "thinking/reflecting" to describe the activity. This difference may be due to the "non-division" of roles. In other words, in $\mathrm{WaSc}$, the children were more prone to share their thoughts since they were all doing all actions together. In contrast, in WoWc, each child had an individual task for the benefit of the group, and hence, describing only the virtual elements or regulating their reciprocal interactions may be enough.

\section{DISCUSSION}

The present study highlights that the WoW and WaS paradigms influenced how the children understood and related to the virtual contents, the physical world, and how they organized social interactions. These findings are partially consistent with the results of previous studies on the WoW and WaS paradigms. In the following sections, we will first shortly discuss the consistencies and divergences between our current research and previous research on the WoW and WaS paradigms. Subsequently, we will use this analysis to derive distinctions between $\mathrm{WoW}$ and $\mathrm{WaS}$ experiences and propose a framework to inform practitioners on adequate AR/MR solutions for their mobile applications.

\subsection{A broader perspective on the potential and limits of the WaS and WoW paradigms}

The results of our study show that the WaS paradigm supported: (1) a more holistic understanding of the experience; (2) a higher attention on the physical world; and (3) a more horizontal way of organizing social interactions compared to the WoW paradigm. Furthermore, they highlight that the WoW experience tended to concentrate most of the children's attention on the screen of the device. These results are partially consistent with our previous study $[10]$ and with research on the potential and limits of the two paradigms. Specifically, in our previous research, we found that children that used the WaS to create fictional stories were more prone to use the physical world as a narrative element in their stories and to co-create shared narratives, in comparison to children who used to WoW. Similarly, our current findings are consistent with the research carried out by Müller et al. [14], which showed that people using the WoW tended to mainly focus on what happens on the device (screen), without paying attention to their surroundings. These consistencies offer a robust background to claim the role of these two paradigms in directing users' attention toward different elements of the experience (e.g. the context or the device) and in shaping social interactions. 
Consequently, they offer relevant guidelines for designers and researchers who want to define optimal AR/MR solutions for their mobile applications.

Nonetheless, despite these consistencies, we also found some relevant differences between our current research and our previous studies regarding the WaS paradigm. Specifically, in one of our previous study [10] we observed that children using the WaS tended to actively explore the physical world with the projection, and physically interact with it (e.g. exploring different projection surfaces; touching / playing with the projected image; moving elements in the physical world to allow a better fit of the digital content; etc.). However, in our current research, we did not observe any instance of experimentation or interaction with the physical world or with the projected image. These differences can be due to the kind of task proposed and to the configuration of the system. In the current study, children were involved as spectators of a pre-given narrative. This means that all contents were already created and children just need to discover them. To this end, we have chosen to use markers in the implementation of the WaS to associate easily and quickly the virtual content to a specific location. In contrast, in the previous study, children were allowed to freely create their stories and the WaS operated in a very low fidelity system configuration which actually did not automatically associate the virtual content with a specific location, but rather allowed the children to freely locate the digital content in the physical location that they felt was relevant for that content.

We observed similar results in a cultural heritage project called Refugi 307 [20]. In the first design iteration, we used also a low-fidelity prototype without physical markers. Hence, the virtual content was not located automatically, but the children were given the possibility of interacting with each content only in specific locations. The content was predefined and specifically designed for the guided visit that a group of guides perform for school classes. Despite this, the children performed a good deal of interactions with the augmented content such as spontaneously embodying situations that civilians during the Spanish Civil War had lived in the shelter. For example, during an activity when a bomb raid was projected, one child cringed when a virtual bomb exploded. Another child tried to interact with the displayed content of a still projected image of a power generator by enacting the action of turning it off using its on/off switch. In contrast, in the second design iteration, in which we started using physical markers, the children hardly performed any of these actions and only interacted with the augmented content when the visit guide encouraged them to do so.

These two cases show how the presence or the absence of markers strongly shapes the ways of using, acting, and making-sense of the physical world. Specifically, in the present study, we observed that the markers shaped the mobility patterns of children; they limited the possibility of interacting with the physical space; and they transformed the space into discrete units, in which, what mattered in the experience occurred only at the location of the marker.

We can therefore hypothesize that the absence of markers allows children to consider all spaces as potentially valid for exploration, play and interaction. This difference, combined with the aforementioned potential and limitations of the WoW and WaS paradigms, offer a meaningful starting point to discuss the articulations between the two AR/MR paradigms and marker-based or marker-less solutions.

\subsection{An initial conceptual framework for AR/MR design for children}

The results of the current study, complemented by the outcomes of our previous studies, allow us to build initial distinctions between the strengths, weaknesses and potential of the two AR/MR paradigms and their articulations with marker-based or marker-less solutions. The goal of this analysis is not to provide a definitive and complete framework, but to build a form of intermediate-level knowledge [2] that can help practitioners in taking design decisions (and defining research questions) to support specific kinds of play and learning experiences in AR/MR technologies for children. A visual description of this initial framework is illustrated in Figure 10.

The proposed model is inspired by the principles of the semiotic square [6]. In the four corners, we depict the different design choices (WoW Marker-based; WaS Markerbased; WoW Marker-less; WaS Marker-less) as discussed previously in this paper. This structure is subsequently used to plot the role of the different design choices in: (1) shaping social interactions (horizontal axis); (2) supporting different kinds of experiences (vertical axis); and (3) directing users' attention toward different elements of the experience (quadrants).

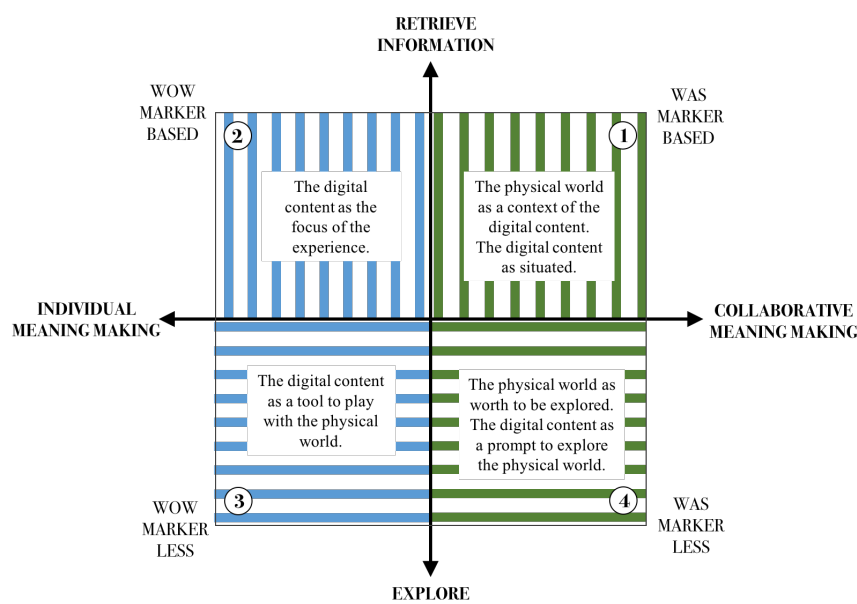

Figure 10. AR/MR Design Framework analyzing WoW and WaS paradigms and marker-based or marker-less solutions

The horizontal axis

The horizontal axis encompasses systems that are either 
based on the WoW or WaS paradigm. Specifically, the $2^{\text {nd }}$ and $3^{\text {rd }}$ quadrants (color blue) describe systems based on the WoW paradigm that either use or do not use markers. Similarly, the $1^{\text {st }}$ and $4^{\text {th }}$ quadrants (color green), describe marker-based or marker-less systems based on the WaS paradigm. As our results suggested, this distinction (WoW/ WaS) strongly shaped the kind of social experience fostered by the system, independently of whether the experience is based or not on markers. Specifically, the children in the WoWc tended to establish a clear role division to solve the task. Instead, the children in the WaSc showed many more instances of shared construction of meaning without assigning a specific role to each one of them. Hence, we suggest that WoW-based solutions can be more suitable to support experiences that require individual meaning-making processes or that presents collective tasks that are not strictly collaborative but depend on the sum of each participant's work. An example could be an augmented reading book for individual amusement. Instead, we suggest that WaS-based solutions could be more appropriate to support collaborative and horizontal meaning-making processes that require a shared construction of knowledge or co-creation processes, i.e. collaborative learning activities.

The vertical axis

The vertical axis encompasses systems that either use or do not use markers to enable AR/MR. Hence, the 1st and 2nd quadrants (vertical lines) describe marker-based WaS or WoW systems. Similarly, the 3rd and 4th quadrants (horizontal lines), describe WoW or WaS marker-less systems. As we observed from the comparison between the current study and the previous ones $[10,20]$, the presence or absence of markers strongly shapes children's patterns of mobility and their way of interacting with space. Specifically, while in marker-based experiences children just move from marker to marker to retrieve the contents, in marker-less experiences children are much more prone to explore the space and the play possibilities of the system. These distinctions suggest that marker-based solutions may be more apt for experiences where users need to focus on specific physical spots. Instead, marker-less solutions can be more apt for free, explorative and creative activities. Nonetheless, since our study did not focus specifically on analyzing this dimension, we need further research to validate this guideline.

\section{The quadrants}

Finally, the articulation of these two dimensions (WoW/WaS; Marker-less/Marker-based) allows identifying four quadrants that offer a glance on the way in which the different design solutions mediate users' attention toward different elements of the experience. As we observed in the current study, systems that belong to the 1st quadrant (WaS and markers) offer a configuration that allows children to pay more attention to the physical context of the experience, despite not prompting specific forms of exploration and interaction with it. Therefore, we suggest that this configuration may be particularly suitable to facilitate experiences aimed at presenting contents that are strongly related and contextualized within the specificities of the physical world but that do not require exploration or interaction with it. For example, AR/MR systems to augment the visit of a museum.

Instead, the 2nd quadrant, by encompassing systems that use WoW and markers, tend to encourage children to mainly focus on the digital content and the device, while paying little attention to their surroundings. This configuration may result particularly suitable to support experiences where the digital content should be the main focus of the task while the physical world mainly acts as a secondary material support. For example, when using AR/MR to access additional digital content such as hidden games in a cereal box or in other promotional material.

On the other hand, the 3rd quadrant represents systems that use the WoW but do not depend on marker recognition. By contrasting the results of our previous research [10] with the current study, we suggest that this configuration may result particularly suitable to facilitate experiences where the digital content can be used to play with the physical world in a layering fashion (e.g. augmented photo booth applications that allow children to use AR to "dress-up" in fun ways).

Finally, the 4th quadrant, represents systems that use the WaS without markers. From the general results of the research project, we suggest that this configuration offers affordances that make it particularly suitable to design experiences that aim at promoting contextual awareness, an active exploration of the physical world, and use the digital content as a prompt to play and interact with the physical world.

Summing up, these different possibilities show how the different design choices of AR/MR solutions cover a fundamental role in mediating children's experience in the relation between physical and digital worlds with mobile technologies. Although further research is still needed to validate these guidelines, we suggest that they may constitute a valuable starting point to guide design researchers and practitioners in the field.

\section{CHILDREN'S PARTICIPATION}

The study was held at a local school in Barcelona. We initially carried out a meeting with the teachers to explain the project. The teachers selected the participating classes and we sent information about the project and the informed consent to the parents or legal tutors. The parents were also invited to phone us for further information. The children participated in the study only if they have the informed consent signed. Furthermore, we explained to them that, if they did not like the activity or did not want to participate they were free of abandoning it.

\section{ACKNOWLEDGEMENTS}

We thank all children who have participated in the study. Supported by the Spanish Ministry of Economy and Competitiveness (Grant TIN2014-60599-P). 


\section{REFERENCES}

1. Nash Anderson, James Steele, Lise Ann O'Neill, and Lesley A. Harden. 2017. Pokémon Go: Mobile app user guides. British Journal of Sports Medicine 51, 1505-1506. http://doi.org/10.1136/bjsports2016-096762

2. Wolmet Barendregt, Olof Torgersson, Eva Eriksson, and Peter Börjesson. 2017. IntermediateLevel Knowledge in Child-Computer Interaction: A Call for Action. Proceedings of the 2017 Conference on Interaction Design and Children IDC '17: 7-16. http://doi.org/10.1145/3078072.3079719

3. Liam Betsworth, Huw Bowen, Simon Robinson, and Matt Jones. 2014. Performative technologies for heritage site regeneration. Personal and Ubiquitous Computing 18, 7: 1631-1650. http://doi.org/10.1007/s00779-014-0766-3

4. Adrian Clark and Andreas Dünser. 2012. An interactive augmented reality coloring book. IEEE Symposium on $3 D$ User Interfaces 2012, 3DUI 2012 - Proceedings, 7-10. http://doi.org/10.1109/3DUI.2012.6184168

5. J. Benjamin Gotow, Krzysztof Zienkiewicz, Jules White, and Douglas C Schmidt. 2010. Addressing Challenges with Augmented Reality Applications on Smartphones. Mobile Wireless Middleware, Operating Systems, and Applications - Third International Conference, Mobilware 2010, 129143. http://doi.org/10.1007/978-3-642-17758-3_10

6. Louis Hébert. The Semiotic Square. Retrieved January 17, 2018 from http://www.signosemio.com/greimas/semioticsquare.asp

7. Tien Chi Huang, Chia Chen Chen, and Yu Wen Chou. 2016. Animating eco-education: To see, feel, and discover in an augmented reality-based experiential learning environment. Computers and Education 96: 72-82. http://doi.org/10.1016/j.compedu.2016.02.008

8. Carey Jewitt. 2013. Multimodal methods for researching digital technologies. SAGE Handbook of Digital Technology research: 250-265. http://doi.org/10.4135/9781446282229.n18

9. Gunther Kress. 2010. Multimodality. A social semiotic approach to contemporary communication. Routledge, London, UK.

10. Laura Malinverni, Julian Maya, Marie-Monique Schaper, and Narcis Pares. 2017. The World-asSupport: Embodied Exploration, Understanding and Meaning-Making of the Augmented World. Proceedings of the 2017 CHI Conference on
Human Factors in Computing Systems (CHI '17)., ACM.

http://doi.org/http://dx.doi.org/10.1145/3025453.30 25955

11. Laura Malinverni, Joan Mora-Guiard, and Narcis Pares. 2016. Towards Methods for Evaluating and Communicating Participatory Design: a Multimodal Approach. International Journal of HumanComputer Studies 94, C: 53-63. http://doi.org/10.1016/j.ijhcs.2016.03.004

12. Paul Milgram. 1994. Augmented Reality: A class of displays on the reality-virtuality continuum. SPIE, Telemanipulator and Telepresence Technologies 2351: 282-292.

13. David Molyneaux, Shahram Izadi, David Kim, et al. 2012. Interactive environment-aware handheld projectors for pervasive computing spaces. Lecture Notes in Computer Science (including subseries Lecture Notes in Artificial Intelligence and Lecture Notes in Bioinformatics), 197-215. http://doi.org/10.1007/978-3-642-31205-2_13

14. Jens Müller, Roman Rädle, and Harald Reiterer. 2016. Virtual Objects as Spatial Cues in Collaborative Mixed Reality Environments: How They Shape Communication Behavior and User Task Load. Proceedings of the 2016 CHI Conference on Human Factors in Computing Systems (CHI '16)., ACM, New York, NY, USA, 1245-1249.

http://doi.org/http://dx.doi.org/10.1145/2858036.28 58043

15. Daniel Pacheco, Sytse Wierenga, Pedro Omedas, et al. 2015. A location-based Augmented Reality system for the spatial interaction with historical datasets. Digital Heritage 1, October: 393-396. http://doi.org/10.13140/RG.2.1.3957.4487

16. Kasey Panetta. Top Trends in the Gartner Hype Cycle for Emerging Technologies, 2017. Retrieved from https://www.gartner.com/smarterwithgartner/toptrends-in-the-gartner-hype-cycle-for-emergingtechnologies-2017/

17. Sara Price and Carey Jewitt. 2013. A multimodal approach to examining "embodiment" in tangible learning environments. Proceedings of the 7 th International Conference on Tangible, Embedded and Embodied Interaction - TEI '13: 43-50. http://doi.org/10.1145/2460625.2460632

18. Gervais Renaud, Jérémy Frey, and Martin Hachet. 2015. Pointing in Spatial Augmented Reality from 2D Pointing Devices. Human-Computer Interaction: $381-389$.

19. Mona Sakr, Carey Jewitt, and Sara Price. 2016. Mobile Experiences of Historical Place: A 
Multimodal Analysis of Emotional Engagement. Journal of the Learning Sciences 25, 1: 51-92. http://doi.org/10.1080/10508406.2015.1115761

20. Marie-Monique Schaper, Maria Santos, Laura Malinverni, Juan Zerbini Berro, and Narcis Pares. 2018. Learning about the Past through Situatedness, Embodied Exploration and Digital Augmentation of Cultural Heritage sites. International Journal of Human-Computer Studies. http://doi.org/10.1016/j.jijcs.2018.01.003

21. Karl D. D. Willis, Ivan Poupyrev, and T Shiratori. 2011. Motionbeam: a metaphor for character interaction with handheld projectors. Proceedings of the SIGCHI ..., 10.

http://doi.org/10.1145/1978942.1979096

22. Karl D. D. Willis, Takaaki Shiratori, and Moshe Mahler. 2013. HideOut : Mobile Projector Interaction with Tangible Objects and Surfaces. Proceedings of the 7th International Conference on Tangible, Embedded and Embodied Interaction (TEI '13), ACM.

23. Karl D D Willis, Ivan Poupyrev, Scott E. Hudson, and Moshe Mahler. 2011. SideBySide: Ad-hoc Multi-user Interaction with Handheld Projectors. In Proceedings of UIST '11, 431-440. http://doi.org/10.1145/2047196.2047254

24. Fabio Zünd, Mattia Ryffel, Stéphane Magnenat, et al. 2015. Augmented Creativity: Bridging the Real and Virtual Worlds to Enhance Creative Play. SIGGRAPH Asia 2015 Mobile Graphics and Interactive Applications: 21:1--21:7. http://doi.org/10.1145/2818427.2818460

25. The fantastic flying book of Mr. Morris Lessmore. Retrieved January 17, 2018 from http://morrislessmore.com

26. Aug That. Retrieved January 17, 2018 from http://augthat.com/

27. Retrieved January 17, 2018 from www.aurasma.com 